Archives de sciences sociales des religions L'héritage des Formes

\title{
Sociologie religieuse et science des religions
}

\section{Gabriel Le Bras}

\section{(Q) OpenEdition \\ Journals}

\section{Édition électronique}

URL : http://journals.openedition.org/assr/23020

ISSN : 1777-5825

Éditeur

Éditions de l'EHESS

\section{Référence électronique}

Gabriel Le Bras, « Sociologie religieuse et science des religions », Archives de sciences sociales des

religions [En ligne], L'héritage des Formes, mis en ligne le 18 juillet 2011, consulté le 01 mai 2019. URL: http://journals.openedition.org/assr/23020 


\section{Sociologie religieuse et science des religions}

In: Archives des sciences sociales des religions. N. 1, 1956. pp. 3-17.

Citer ce document / Cite this document :

Le Bras Gabriel. Sociologie religieuse et science des religions. In: Archives des sciences sociales des religions. N. 1, 1956. pp. 3-17.

http://www.persee.fr/web/revues/home/prescript/article/assr_0003-9659_1956_num_1_1_1933 


\title{
SOCIOLOGIE RELIGIEUSE ET SCIENCE DES RELIGIONS
}

\begin{abstract}
LA revue dont voici le premier numéro a une brève préhistoire; elle naît à l'instant favorable où les sciences comblent la sociologie religieuse de leurs dons ; notre espoir est qu'elle leur portera un contre-don. Evoquer la genèse de notre entreprise, les apports récents des disciplines qui la soutiennent, les services que nous souhaitons de rendre par juste échange : la matière et la forme nous sont comme imposées d'une préface que nous dédions à tous les hommes curieux du sacré, du profane et de leurs liaisons.
\end{abstract}

\section{I}

Dès sa création par le C.N.R.S., en 1945, le C.E.S. a constitué une section de sociologie religieuse. Sans interruption, des cours, des colloques ont réuni une élite de jeunes chercheurs, au siège mobile de la maisonmère (1). Jusqu'au jour où le Conseil d'administration de la Fondation nationale des Sciences politiques a bien voulu nous accorder, avec une générosité exemplaire, le local indispensable à toute régulière activité (2).

Dès son origine, le Groupe de Sociologie des religions, animé par Henri Desroche et François Isambert, a spontanément résolu de rassembler dans un ordre méthodique les études dispersées ou épuisées de son doyen d'âge. Deux volumes ont ainsi augmenté la bibliothèque de sociologie contemporaine (3).

(1) Rue de Montpensier et boulevard Arago. Les conditions de ces débuts furent, malgré la bonne volonté du C.N.R.S., assez inconfortables. Conséquence de la crise du logement.

(2) Le siège de notre groupe est au $n^{\circ} 30$ de la rue Saint-Guillaume, dans un immeuble récemment aménagé par la Fondation : c'est là que nous recevrons avec plaisir la visite de tous ceux qu'intéresse notre revue et tout notre travall.

(:3) Etudes de sociologie religieuse, tome $\mathrm{I}^{\mathrm{er}}$ : Sociologie de la pratique religieuse dans les campagnes françaises; tome 11 : De la morphologie a la typologie. Presses universitaires, Paris, 1955 et 1956. 
Aussitôt commença une seconde expérience: la composition d'un fascicule de Current Sociology demandé par l'U.N.E.S.C.O. Pendant six mois, des fiches furent accumulées, triées, classées. Sur 4.500, environ 900 ont été retenues pour l'impression. Aucune d'entre elles ne porte date antérieure à 1940 et il a fallu choisir dans le lot des quinze dernières années : la récolte dépassait de beaucoup nos prévisions et aussi les proportions de l'élégant fascicule où nous devions la loger (4).

Les travaux de notre commission préparatoire mirent en évidence trois vérités simples. D'abord, la dispersion et le fragmentaire de la bibliographie, on pourrait même écrire son inexistence, puisqu'aucun périodique ne la rassemble et qu'il faut la cueillir par grappes ou unités dans des listes hétérogènes ou des bulletins critiques (5). A fortiori, point de chronique : congrès, recherches, annonces, nous en avons quelques échos de bouche à oreille ou par le hasard des lectures. Ce que nous savons mieux, c'est nos commencements : l'existence de rapports, mémoires pour diplômes, thèses de doctorat dont la polycopie garantit le secret (6).

Ainsi se justifie notre triple désir : imprimer ou résumer les ouvrages de mérite que la rigueur des temps condamne à la clandestinité; stimuler la recherche, l'entraide, la curiosité par une chronique abondante et des notes suggestives; fournir chaque semestre un relevé aussi complet que possible de tous les ouvrages et articles à peine sortis des presses.

Notre projet rencontra aussitôt des adhésions et des encouragements. Le Comité Directeur du Centre d'Etudes Sociologiques l'accueillit avec bienveillance (7). D'Angleterre, d'Amérique nous vinrent des promesses de collaboration (8). Enfin, le C.N.R.S., toujours favorable aux initiatives opportunes, assuma la charge d'une publication qui répondait à tant de vœux (9).

Il apparut tout de suite que, loin d'empiéter sur le terrain d'autrui, nous apportions aux périodiques anciens un complément utile. L'Année sociologique et les Cahiers internationaux de sociologie, embrassant tous

(4) Il parait en méme temps que ce numéro, sous le titre : Sociologie des religions in : La Sociologie contemporaine, 5, 1956, 1, 87 p. (U.N.E.S.C.O.). Nous le désignerons dans ces notes par le terme Bibliographie.

(5) Exception faite pour La Bibliographie internationale de l'histoire des religions, publiée \& Leyde. Signalisation dans notre Bulletin Bibliographique. On y trouve, avec toutes les précisions requises, beaucoup des références données ici et volontairement sous une forme plus sommaire.

(6) Pour la France, des mémoires en vue du diplôme d'études supérieures d'histoire (Facultés des Lettres) ou d'histoire du droit (Facultés de Droit); des mémoires de l'Ecole pratique des Hautes Etudes ; des thèses de doctorat (Droit ou Lettres). Il s'agit presque exclusivement du catholicisme. Voyez dans ce numéro le relevé d'Emile Poulat.

(7) G. Davy, président, G. Friedmann, G. Gurvitch, H. Lévy-Bruhl et moi-même.

(8) Les premières de E. Hughes, directeur de l'American Journal of Sociology et de J. L. Adams, directeur du Journal of Religion et chairman de la Society for the scientific Study of Religion.

(9) M. Dupouy, directeur du C.N.R.S. et M. Lejeune, directeur-adjoint, ont tout de suite redoublé notre ardeur. 
les domaines de la sociologie, ne peuvent consacrer à la religion qu'une part raisonnable de leurs feuillets : leurs directeurs n'ont jamais envisagé de prendre à leur compte aucune de nos trois rubriques (10). En revanche, notre équipe se propose de continuer et d'augmenter sa collaboration à ces deux organes qui ont une si éminente part au progrès de la pensée.

Elle s'honore de prendre place dans une tradition que la France entretient assidûment. Les sciences religieuses ont de profondes racines sur notre sol (11). Pour nous en tenir à l'époque contemporaine, elles sont tout le programme d'une section de l'Ecole pratique des Hautes Etudes dont la contribution a été d'une exceptionnelle richesse (12). Les ouvrages, les bibliothèques se multiplient. Une dizaine de périodiques savants, plusieurs tout fraîchement fondés, sont au service de toutes les disciplines (13). Celle que nous servons ici a sa tradition particulière, que plusieurs écoles, la plus fameuse ayant pour chef Emile Durkheim, ont solidement implantée. Ainsi, nous entrons dans une famille et dans une lignée dont la force nous soutiendra.

Un regard sur les autres pays nous fait encore mieux mesurer nos dépendances et nos appuis. L'Allemagne et les Etats-Unis comptent parmi leurs morts illustres Max Weber et Ernst Trolsch, W. Dilthey et Joachim Wach, pour nous borner à quatre noms (14). Et nous sommes heureux de coopérer avec de nombreux collègues, d'ajouter notre titre à celui de nombreuses revues qui, dans le monde entier, nous donnent l'exemple d'un travail sainement et sereinement organisé (15).

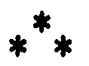

Par ces déclarations préalables, les caractères de notre revue sont insinués. Il convient de les définir et commenter, pour éviter les équivoques dont les sciences humaines et surtout les sciences religieuses sont encombrées.

(10) L'Année sociologique, fondée par Durkheim, fournit dans une section Sociologie religieuse, dont nous avons l'honneur d'assumer la responsabilité, l'analyse d'un certain nombre d'ouvrages. Elle publie, en outre, des articles de sociologie religieuse. Les Cahiers internationaux de Sociologie font, eux aussi, une bonne place à la sociologie religieuse, articles et comptes-rendus. Nous n’aurons qu'à compléter une littérature scientifique déjà riche et en plein essor.

(11) L'Etude comparée des religions du P. PINARD DE LA Boullaye fait implicitement ressortir cette vérité.

(12) Fondée en 1886, l'Ecole compte aujourd'hui 24 directeurs d'études, qui enseignent l'histoire et le système de toutes les grandes religions, mortes ou vivantes. La série des Annuaires contient le résumé des cours. Une Bibliothéque accueille les ouvrages des maítres et des dipló-
més.

(13) Revue d'Histoire des Religions, Revue des sciences religieuses, Année théologique, Année canonique, Revue de droit canon, La Maison-Dieu, Revue d'histoire de l'Eglise de France, Revue d'histoire et de philosophie religieuse, Recherches de science religieuse, etc...

(14) J. WACH, dans La Sociologie au $X X^{\mathrm{e}}$ siècle (ouvrage publié sous la direction de G. Gurvitch) a fait un bilan assez général.

(15) Notamment American Journal of Sociology, American Sociological Reviero, Archiv für Religionswissenschaft, British Journal of Sociology, Journal of Religion, Kolner Zeitschrift für Soziologie, Sociological Review, etc... 
Bien que la plupart des fondateurs aient consacré leurs recherches au catholicisme, notre vue embrasse toutes les religions, vivantes et mortes, archaïques ou modernes. Nous avons sollicité, obtenu le concours de savants appliqués à l'étude de l'Islam, du bouddhisme, des polythéismes, notre but étant la comparaison.

Nous croyons nécessaire la coopération de toutes les sciences. Les controverses de frontières nous répugnent. Ethnologues, psychologues, voire mathématiciens ont leur fort, d'où ils peuvent déjà nous aider, mais en outre, ils partagent plusieurs de nos terres et enfin ils appartiennent à l'unique caravane de la découverte scientifique.

Des hommes de toutes croyances et incroyances voisinent dans le cortège et nous n'avons point à connaître leurs sentiments. Cette revue ne saurait être au service d'aucune doctrine, confessionnelle ou anticonfessionnelle. Elle accueillera l'exposé paisible de toutes les recherches de toutes les théories, avec le souci exclusif de servir la science.

Supposer que tous les sociologues appliqués à l'étude des relig'ons se mettront jamais d'accord sur le domaine, le but, les méthodes de leur recherche, serait avouer un optimisme enfantin ou une résignation sénile. Les sciences vivantes se reconnaissent à l'ambition d'étendre leur champ, à la variété des espérances qu'elles suscitent, au renouvellement constant des méthodes. Pour justifier cette exubérance, il suffit qu'elles spécifient leur objet. La condition se réalise dès qu'une définition rallie presque tous les suffrages : nous obtiendrons sans doute la quasi-unanimité sur une formule simple: la sociologie des religions se propose d'étudier la structure et la vie des groupes organisés dont le sacré est le principe et la fin (16). Dans cette phrase brève apparaissent trois mondes : le communiel, assemblée des adhérents ; le supranaturel, où siègent les puissances cachées ; le civil, au milieu duquel s'établit la compagnie (17). C'est le premier de ces mondes qui intéresse principalement la sociologie : elle décrit et tâche d'expliquer sa composition et sa cohérence; de mesurer ses forces et ses déficiences; de comprendre ses relations avec les deux autres mondes, l'image qu'il se forme d'eux et de lui-même.

Ainsi, le sociologue n'entreprend-il d'explorer seul ni les mystères du supranaturel ni les maquis de la cité terrestre. Il accompagne théologiens et liturgistes, canonistes et légistes pour comprendre les liaisons, les projections, les langages. Son office est de discerner les structures, de saisir la vitalité des groupes humains, à la lumière de toutes les sciences de

(16) Il va de soi que nous ne séparons pas radicalement vie et structure, que nous n'ignorons point les connexions du sacré (dont le sens se discute) avec le profane : mais nous soulignons l'importance des mesures de la vitalité, programme valable pour toutes les religions.

(17) Nous avons donné les justifications de ce tripartisme dans le tome I (Prolégomènes) d'une Histoire du droit et des institutions de l'Eglise en Occident, Paris, 1955. 
l'homme. Il ne refait point leur ouvrage et il leur abandonne, s'il y croit, le pur individuel. Toutes les manifestations collectives et cohérentes de la vie religieuse lui appartiennent (au moins par indivis) soit qu'elles se fixent en institutions soit qu'elles traduisent instantanément la vigueur (ou la faiblesse) d'un groupe.

La tendance des philosophes, qui ont une part si éminente dans la naissance et le développement de la sociologie des religions, est aux théories générales, que semble autoriser un choix intelligent d'observations. Nous avons toujours recommandé, quant à nous, (et il est juste d'écrire : avec leur plein consentement) une division du travail poussée à l'extrême, la construction d'une pyramide dont la base est au ras du sol des paroisses ou des tribus, au niveau des légendes, aux confins de la magie. On nous pardonnera de rappeler ces positions élémentaires (18).

Chacune des religions devrait être étudiée, dans le temps et dans l'espace, depuis celle des groupes Yirkalla jusqu'au christianisme. On observerait leur développement continu, leurs nuances locales, avec le concours des ethnologues et des psychologues, des historiens et des géographes, des théologiens et des canonistes, de tous les auxiliaires qualifiés, qui seraient des serviteurs maîtres. Car il s'agirait d'une suite d'examens "spécialisés " et coordonnés, portant sur les croyances, les rites, les disciplines et où l'on distinguerait avec soin (sans les séparer) le construit et le vécu, disons le système et les réalités (19).

Le sociologue progresserait par degrés prudents, s'astreignant aux plus humbles besognes : récolte des récits, entretien des sages, lecture des traités, interprétation des cérémonies; puis observant les réalités : statistique des monuments et des actes, cartographie et monographie, sémiologie et psychologie des fidèles en des villages et des quartiers représentatifs (20). L'étude du groupe civil lui révèlerait la place du groupe religieux au sein du village ou de la ville: confusion, dans l'Islam; intimité, en pays chrétien; diaspora, chez les peuples laïcisés. La relation entre les deux mondes visibles, l'attraction de l'invisible suggèreront une typologie, une étiologie, une discrète nomologie, dont la perfection serait à la pointe hypothétique de l'audacieuse pyramide.

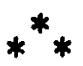

Des sceptiques se décourageront, des impatients refuseront le "planisme ", des dogmatiques s'étonneront de ne point reconnaître leur refrain : dans ce chour dissonant, en sera-t-il un seul qui n'ait encouragé notre espérance par son apport, plus précieux que tous nos programmes?

(18) Présentées moins succinctement dans L'Anni? Sociologique, 194.8-1949 (éd. 1952), pp. 287-294.

(19) Dualisme qui progresse avec la civilisation. Chez les peuples archaiques, le mythe et le rite sont vécus, dans l'élite intellectuelle du christianisme, la distinction s'accentue entre la pensée et l'action, que les vrais croyants cherchent à coordonner.

(20) Voyez notre préface à la Bibliographie. 
Pour nous en tenir aux dix dernières années, rappelons quelques-uns des progrès les plus notables des sciences de la religion et des diverses branches de la sociologie profane (21).

L'histoire des religions a dressé plusieurs fois son bilan, occasion excellente de l'améliorer (22). T'outes les religions de l'Antiquité, toutes les religions vivantes: archaïques ou savantes, tribales ou universelles, toutes les sectes remuantes ont livré à de pénétrants érudits le dessin de leurs transformations (23), le détail de quelques cultes (24), parfois des énigmes sur leurs origines (25). Chacun des secteurs: théologie, liturgie, discipline, s'enrichit d'éditions critiques (26), de chapitres originaux, d'esquisses provisoires du développement (27).

Entre les religions, entre les secteurs fleurit la comparaison, qui suggère emprunts mutuels ou réponses indépendantes et typiques à d'identiques problèmes (28). La voie s'ouvre ainsi aux sciences les plus générales : phénoménologie du mythe ou de tout l'appareil religieux (29); psychologie de la personne et des communautés (30); ethnologie, qui enveloppe toute la vie des groupes, depuis les formes de la parenté jusqu'aux rites de la chasse, depuis les incantations du sorcier jusqu'aux extases du chaman (31).

Cependant, toutes les branches de la sociologie croissent et surgeonnent. La naissance, l'extension, la reconstruction des villes font

(21) Nous nous bornons à choisir des exemples et nous abrégeons les références à des ouvrages et articles connus de tous nos lecteurs, ou faciles à retrouver. (Table de la Bibliographie).

(22) Mortier-Gorce, coll. Mana, Christus und die Religionen der Erde, Brillant-Aigrain.

(23) Transformation de la piété en Egypte, avec le régime politique (Drioton, Dévotion privée, 1949), développement du brahmanisme (Revou, L'Inde classique, 1949), préhistoire de l'Islam (Philey, Background of Islam, 1947 ; RYckmans, Religions arabes préislamiques, 1951), de la religion grecque (Roux, Argonautes, 1949); rupture entre magie et religion (WeBsTer, Magic, 1942), entre orthodoxes et sectes (Desroche, Année sociologique, 1952, éd. 1955).

(24) Thèse de Schilling sur Vénus, 1855.

(25) Manuscrits de la Mer morte.

(26) Que de suggestions dans le Pontifical romain et les Ordines, édités par M. Andrieu.

(27) Au cours du dernier lustre, ont paru (ou commencé de paraître) plusieurs histoires de la liturgie (Righetti) et du droit canon (Feine, Plochl, Le Bras).

(28) Theologie comparé : GARDET et ANAwaTl pour l'Islam (Introduction à la théolngie musulmane, 1948). Convergence de l'archéologie et de la liturgie : Lassus, Sanctuaircs, 1847).

(29) Travail facilité par les dictionnaires de Grimal, de Lavedan. Etudes de van der Leeuw, d'Eliade (1951), d'Hirschmann (1952). Examen historique des divers mythes (SÉcuan, Prométhie, 1051) qui, jusque chez des peuples archaiques, pourraient recéler une métaphysique (Griaul.s, Dieu d'eau, 1948). Dans la Revue internationale des droits de l'Antiquité, 1955, p. 39-106, Denise Cocquerillat donne un exemple remarquable d'institutions concordantes : Les prébendes patrimoniales dans les temples à l'époque de la $1^{\text {re }}$ dynastie de Babylone " (paralleles aux bénéfices ecclésiastiques).

Voyez la bibliographie des Croyances et représentations dans la Bibliographie, p. 509-527.

(30) M. LeEnhardt, Do Kamo, 1947. G. Dieterden, La religion Bambara, 1951.

(31) Cette quadruple érocation fait surgir chez nos lecteurs les noms de Cl. Lévi-Strauss et de K. Young, de J. Aymard et d'Evelyne Lot-Falck, de Contenau et de Wagner, d'Eliade et de Griaule. - Ni au début ni au terme de tous ces travaux, l'accord n'est unanime sur le sens de la religion, ses rapports avec la magie, ni même sur les fonctions de chacune des sciences qui s'appliquent à son étude. 
prospérer la sociologie urbaine tandis que la sociologie rurale tardivenent se hâte d'observer des champs qui se dépeuplent (32). Travail et loisirs, langues et arts, droit et morale, tout ce qui regarde la structure et la vie des sociétés appelle l'attention d'enquêteurs et de théoriciens, très souvent, par fortune, de savants qui cumulent ces deux vocations (33).

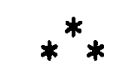

Reprenons le schéma des trois mondes que la religion coordonne, pour relever quelques progrès accomplis depuis dix ans dans les conclusions de la sociologie religieuse et aussi dans les voies de la recherche.

L'attention s'est portée sur la structure et la vie autonomes des plus grandes et des plus petites sociétés religieuses. Epreuves et entreprises du christianisme et de l'Islam ont suscité l'analyse de deux organisations universelles : composition et coordination, activités et potentiel humain, éléments de la puissance spirituelle et temporelle (34). Cependant que les peuples archaïques, dont les coutumes sont menacées et les sectes qui prolifèrent sans ralentissement gardent et attirent dans leur domaine restreint des enquêteurs nombreux (35). Des phénomènes communs appellent un redoublement des études : affaiblissement des masses de pratiquants, crise de l'autorité, reflexion sur les sacerdoces (36).

Peut-être les progrès sont-ils plus visibles dans l'étude des relations entre sociétés religieuses et sociétés civiles : ce qui s'explique par le caractère beaucoup plus actuel et pratique de cette dimension. Les peuples qui passent promptement de la civilisation du fétiche à celle de l'atome, et même les peuples découvreurs de l'atome imposent à leurs

(32) Villes et campagnes, publié sous la direction de G. Friedmann, à l'issue de la Semaine sociologique de 1954, résume les problèmes et leurs rapports avec la religion, que l'on pourrait approfondir en lisant les ceuvres cataloguées à la Bibliographie de sociologie religieuse, $n^{0}$ 747790 .

(33) Faut-il souligner l'apport indirect (conn issance de la civilisation) ou direct (connexité avec les religions) de tous ces sociologues appliqués à des études profanes ? Ce que la linguistique apporte à la sociologie religieuse, G. I)umézil nous en est témoin et les juristes philosophes ont tôt fait de rencontrer la croyance au sacré.

(34) Un simple coup d'oil sur la Bibliographie montrera cette avance exceptionnelle. Le judaïsme et surtout les grandes religions de l'Asie appellent beaucoup de sociologues. Et nous souhaitons le concours des historiens de religions mortes ou vives. - Dans la sociologie du catholicisme, progrès des critères d'appartenance (notion de marginalité) qui devraient être approfondis pour toutes religions.

(35) Ceux que l'on appelait naguère les primitifs, et qui ont fourni à Durkheim, à Lévy. Bruhl leurs principaux themes, continuent de susciter observations et réflexions qui éclairent les rapports entre religicn et nagie, mystique et empirisme.

Sectes, ecclésioles, utopies deviennent un des grands domaines de la sociologie religieuse. Voyez, outre la Bibliographie, un article de H. D. (Desroche) . Sectes, utopies, et modes de coopération „ dans L'Année sociologicrue, 1952 (éd. 1955).

(36) Recul général constaté par le P. Naïdenoff, analysé par le P. Desqueyrat, par Lce, par Müller. Réflexions théoriques (Congar) ou pratiques (U'Brien) sur le sacerdoce. L'évolution contemporaine du concept d'obéissance du clergé et des fidèles dans le christianisme mériterait plus d'un volume.

Tensions intérieures : pour l'Islam, GrbB, Modern Trends, 1949 ; Fernav, Réveil, 1953. 
communautés religieuses soit un choc mortel soit une réadaptation des structures et des activités. Entre révolution et réforme, il y a place pour tous les degrés de la contamination, de l'acculturation. Quand les barrages noient les villages ; quand les missionnaires parcourent les savanes perdues ; quand les fils des anthropophages se mettent à dévorer Bergson, les sociétés "animistes ", islamiques ou chrétiennes sont si fortement ébranlées par la juxtaposition des usines à la ferme, du Coran ou des Evangiles au Vaudou et des maîtres de la Sorbonne aux sorciers du hameau que le principal objet de la science est d'observer ces dramatiques bouleversements (37). Encore n'avons-nous fait allusion qu'à des rencontres casuelles. Or, le fait capital de notre temps est la variété des contacts entre sociétés de type ou de croyances diverses. Jamais de telles masses de réfugiés ne s'étaient ébranlées puis stabilisées en Europe et en Asie (38); jamais de telles perturbations politiques n'avaient secoué les disciples du Christ, de Mahomet et de Bouddha (39) ; jamais les institutions dans lesquelles se meuvent les hommes ne subirent de transformations si profondes et si corrosives (40). On ne saurait trop recommander les études scientifiques sur l'influence des migrations, des révolutions et des milieux de vie.

Le temps critique, pour une religion, est celui où elle subit l'action de la société civile plutôt qu'elle ne l'inspire. Mais dans ce retournement, la société religieuse ne perd point toute sa force. Elle s'applique à la reconquête et à la persuasion. Les études récentes sur la politique, la famille, les villes, les campagnes font place au "facteur" religieux, et

(37) Les syncrétismes offrent d'innombrables expériences du contact fructueux et dissolvant. Voyez les observations de R. Bastide au Brésil, de Fernandes en Afrique noire, de Briggs chez les Kmers, d'Escarpit au Mexique, de Lévi-Strauss dans un village Moy, de Métraux dans la vallée de Marbial. Ils supposent un mélange provisoirement actif : l'intégration d'une nouvelle culture peut, au contraire, provoquer une élimination insensible de toutes les anciennes valeurs religieuses. Voyez les communications au 29e Congrès international des Américanistes (1952). La réception contrôlée des Huttérites est probablement un phénomène exceptionnel. Aucune région n'offre présentement un champ d'observation plus passionnant que l'Afrique noire : rencontre de l'Islam avec le fétichisme et le christianisme (Bibliographie, $\mathrm{n}^{\circ} 257-264$; ajoutez P. Alexandre, "L'Afrique noire et l'expansion de l'Islam ", in Le Monde non chrétien, 1955, pp. 315-354); rencontre des plus anciennes formes de civilisation avec les plus modernes.

(38) Nous avons dès 1940 appelé l'attention sur les conséquences religieuses des transports de populations. Ce fut d'abord l'incorporation de tous les hommes valides dans les armées, puis les exodes semi-volontaires, puis les évacuations imposées. Les réfugiés allemands ont inspiré des études pertinentes mais trop rares, tardives et brèves. La séparation de l'Inde et du Pakistan a déplacé 14 millions de personnes : 7 millions de musulmans auraient quitté l'Inde pour le Pakistan et 7 millions de non-musulmans, le Pakistan pour l'Inde : un tel mouvement qui a une cause religieuse, n'a pu être sans conséquences religieuses.

(39) La rencontre de l'Islam et du communisme a été étudiée pour l'U.R.S.S. (Monteil, 1953, et Chambre, 1955) et l'Iran (Miller, 1951). Celle du catholicisme, maintes fois, pour les pays de l'Est européen et sommairement pour l'Inde. Nombreux travaux sur le sort de l'Orthodoxie.

(40) Qu'il s'agisse de la famille ou de l'usine, la sociologie religieuse n'a point pour centre unique les lieux de culte : ses véritables cadres sont fournis par toute la société. C'est par l'étude complète des structures et de la vie d'un peuple, d'une ville, d'un village que nous comprenons les formes et la mesure des sociétés religieuses. En revanche, les religions nous aident à comprendre la morphologie sociale (castes, confréries, etc...) : sujet de nombreuses études, qui sont au centre de notre science. 
souvent une place primordiale (41). Non seulement dans les pays comme les terres d'Islam où l'incrédulité de quelques modernes, l'urbanisation fatale aux pratiques et les relâchements de la discipline ne suffisent point à disjoindre la société politico-religieuse, mais encore dans les démocraties occidentales et jusque dans les démocraties populaires (42). L'irréligion croissante, elle aussi, phénomène de classe autant que de culture, appelle notre attention : elle peut à son tour prendre la forme de dogmes, de cultes, de morales, devenir une religion du salut temporel (43).

Toutes ces actions s'accompagnent d'idéaux ou d'idéologies que le sociologue analyse comme l'opinion des groupes sur leur mission. Dans les confessions chrétiennes, les programmes et les institutions se sont multipliées où les élites traduisent leur conception d'une société fondée sur les principes évangéliques (44). L'histoire de ces représentations, écrite avec talent au cours de la dernière décennie, révèle les espoirs et les vœux d'une société religieuse qui professe une doctrine de l'ordre humain.

Moins avancée demeure l'étude des relations avec les mondes supranaturels, qui sont cependant la fin de toute religion. Cependant, le caractère social de la gigantomachie (45); la hantise de liaison dans les anciennes religions de salut (46); le souci de l'unité, manifeste dans le naturisme des " primitifs" (47); l'angoisse des élites, sensible au corpus hermeticum (48), le concordisme de l'Aréopagite (49), les flambées incessantes du prophétisme, du messianisme (50) ont été mis dans une telle lumière que si nous savions nous faire Grecs, Romains ou Bantous, l'organisation de l'Au-delà et ses contacts avec les sociétés humaines nous deviendraient pleinement intelligibles.

Les sciences sacrées elles-mêmes sont soumises à l'examen des sociologues selon le vœu de leurs docteurs. Liturgie, droit canon, morale,

(41) Pour nous en tenir à la France, le rôle des catholiques et des protestants (Schram, Protestantism and Politics in France, 1954) dans notre politique intérieure fait l'objet de nombreuses monographies et synthèses, dont le principal animateur est François Goguel.

Chaque Etat doit compter avec ses sociétés religieuses : le Canada (S.D. Clark) comme Isra ill (R. Bloch). Que les sociétés religieuses pèsent sur les opinions et les options internationales, on tendrait à exagérer plutôt qu'à restreindre cette vérité : le nationalisme (BAroN, Modern Nationalism, 1947) et les Internationales reçoivent quelque aliment des religions. Et les doctrines politiques ne sont pas sans lien avec les aspirations des sociétés religieuses : qu'il s'agisse des partis confessionnels ou de certaines formes du socialisme.

(42) H. Desroche, Signification du marxisme, 1949.

(43) Nécessité d'une sociologie de l'athéisme, entreprise depuis peu.

(44) Sur le catholicisme social en France : Duroselle, Rollet, Hoog; exposé doctrinal dans M.J. Williams (1950) et J. Villain (1953-1954). - Chez les protestants, variété de tendances.

(45) F. Vian, La guerre des géants, 1952.

(46) F. Cumont, Lux perpetua, 1949.

(47) O'Reilly l'aperçoit chez les Polynésiens ; Leenhardt, en Asie et en Afrique.

(48) Festugière, le Dieu cosmique, 1949.

(49) RoQue, L'univers dionysien, 1954.

(50) NEHER, Essence, 1955. 
théologie reçoivent quelque lumière des divers foyers d'une discipline qui naguère les inquiéta (51).

Dans les mythes, les rites, les pratiques de tous les temps que de sujets d'inépuisable méditation s'offrent aux curieux des images, des sentiments, des pensées qui hantent les collectivités humaines, pour honorer, modifier, parfois pour créer les dieux.

La permanence, l'accélération des progrès accomplis dans les acquisitions de la sociologie religieuse sont assurés par le progrès concomitant des méthodes de recherche et des sciences auxiliaires.

Dans sa première phase, toute descriptive, la sociologie - qui n'est encore que sociographie - bénéficie des perfectionnements de la statistique et de la cartographie, de l'école et de l'ethnographie: une connaissance beaucoup plus précise des nombres, une vision spatiale des dispersions et des groupements, le détail de l'environnement, l'inventaire des usages nous révèlent à la fois la société religieuse et la société profane. Morphologie et psychologie des communautés religieuses révèlent toute leur originalité, en même temps qu'apparaît leur insertion dans un monde civil administrativement décrit, catalogué (52).

$\mathrm{Au}$ deuxième palier, où elle mérite pleinement son nom, la sociologie tente d'expliquer les faits révélés par ses enquêtes. Son ambition est favorisée par les apports récents de l'histoire, de l'ethnologie, de la psychologie.

Systèmes et réalités se développent dans le temps. Théologies ou liturgies, pratiques et conduites varient continument. Les recherches sur leurs variations ne relèvent point de la sociologie. Mais sans l'histoire qui les couronne, comment le sociologue discernerait-il l'essentiel et le transitoire, les diverses façons de concevoir l'essentiel lui-même ? Entre le christianisme du second siècle et celui du $\mathbf{X X}^{\mathrm{e}}$, il y a trop de distance pour qu'un Origène sociologue se reconnût sans une heure d'éblouissement dans la société chrétienne d'aujourd'hui. De la masse des faits particuliers, que récolte et interprète l'histoire (le chroniqueur se borne à les aligner), le sociologue tire d'exactes généralités. Sa typologie de l'autorité ou des sectes, des puissances religieuses et profanes, du réseau de leurs rapports internes ou externes, des représentations et des rites, s'enrichit à mesure que l'histoire lui fournit des matériaux bien taillés. Pourquoi remontionsnous jusqu'à Origène? Des savants en pleine vigueur ont renouvelé par leurs études sur le manichéisme ou le jansénisme notre sociologie des dissidences (53).

(51) Qu'on me permette de renvoyer à mes études : Liturgie et sociologie (Mélanges ANDRIEv, 1956) ; Sociologie religieuse et droit canon (Annéc canonique, 1952). Plusieurs de nos références montrent les approches de la théologie morale ou dogmatique.

(52) Les institutions publiques comme l'I.N.E.D. et l'I.N.S.E.E. ne se bornent pas à fournir des renseignements statistiques : ils secondent très efficacement certaines enquêtes de sociologie religieuse.

(53) Il suffira de nommer, parmi mes collègues des Hautes Etudes, Puech et Orcibal. 
Mieux que cette vue organique de la société, l'histoire nous procure l'intelligence de certaines causes. Il ne s'agit point des causes d'un événement particulier - affaire du véritable historien - il s'agit des causes de l'apparition et de la propagation d'un type, à un plus haut degré, de la succession des types. L'hagiographie du bouddhisme, de l'Islam ou du christianisme affine peu à peu notre sociologie de la sainteté, comme l'histoire des monastères ou - trop rare - des lamaseries et des zaouias, notre sociologie du canobium (54); l'histoire des confréries et celle des fondations, notre sociologie des personnes morales et des cités où prospèrent ces substituts des communautés tutélaires (55).

L'histoire, cependant, répond-elle à nos profondes curiosités? Elle nous révèle des causes immédiates, des opérations, des événements : que l'hérésie renforce l'autorité ; que le luxe appelle une organisation de l'ascétisme; que le sommeil d'un groupe cultuel favorise l'exubérance des fraternités et des chapelles (56). Pourquoi ? Le secret, dont une part nous échappe, est dans l'ensemble des activités du groupe et au tréfonds des âmes. Aux ethnologues de nous apprendre ce qui ne reste point mystère : par des inventaires complets, des analyses particulières, voire de séduisants récits de voyages, ils éclairent toute une civilisation (57). Tandis que sous des noms divers, les psychologues franchissent le seuil des consciences, proposant des sentiments collectifs - aussi bien qu'individuels - des interprétations pour le moins suggestives (58).

Une sociologie de la religion romaine, que l'abondance des travaux récents rend possible et désirable illustrerait notre propos, qui est de faire concourir toutes les sciences (sur pied d'égalité) au succès de la sociologie religieuse (59). Sans doute les premiers siècles reverraient-ils entre frères latins des combats sanglants. Mais on s'accorderait sur les caractères du culte : civique et familial, officiel et autoritaire, temporel et formaliste. A la fin de la République, l'épigraphie funéraire nous met en présence du personnalisme, du libéralisme et d'une sotériologie. Ainsi le triple réseau des rapports - communiels, civiques, supranaturels - a totalement changé. L'histoire nous le montre et elle offre des justifications plausibles : les révolutions sociales, les contacts avec l'étranger. Archéologues, ethnologues, linguistes classeront les témoins du changement; psychologie,

(54) Condition sociale et surtout critères de la perfection sont décelés par des islamisants comme Massignon et Dermenghem; dans le catholicisme, par Festugière et Lavelle. - Essai de typologie des ordres religieux par $K$. Francis.

(55) Le Congrès annuel de la Société pour l'histoire des droits de l'Antiquité a, sur notre demande, consacré toutes ses séances en 1954 et 1955, aux fondations. Nous avons eu l'honneur, en 1954, de résumer les rapports a la séance finale et nous nous proposons de tirer prochainement les conclusions sociologiques dont les plus saillantes ont été proposées d'avance par Eberhard F. Brück, Uber romisches Recht im Rahmen der Kulturgeschichte, Berlin, 1954.

(56) Ce sont là des faits sociaux, qu'enregistre avec gratitude le sociologue : il lui reste à parfaire l'explication, à constituer une typologie, à relever les constantes.

(57) Que l'on nous donne, s'il se peut, beaucoup de Tristes tropiques.

(58) L. Gernet, Histoire des religions et psychologie (1954), KardinER, etc..., Psychological Frontiers (1945). BaLandier, Convergences (1948); Mauss, Sociologie et anthropologie (1950).

(59) Je résume ici une conférence faite en 1954 à l'Ecole française de Rome sur l'évolution du sentiment religieux à Rome, d'après les sources juridiques. 
psychopathie, psychanalyse aideront à comprendre les altérations, les substitutions, les syncrétismes que l'histoire des religions découvre adroitement. Les changements de structure et les oscillations de la vitalité se traduisent dans les temples, le matériel sacré, le décor et les usages expressifs d'un mode et d'un niveau d'existence commune, le vocabulaire savant ou courant. Et le sentiment qui soutient tout le système et que le système entretient, subit aussi le choc des angoisses collectives, des images nouvelles du monde, des critiques ou des tentations que les sociétés helléniques, orientales ou africaines portent jusqu'au cœur de la Ville $(60)$.

Les dettes de la sociologie des religions seraient élégamment acquittées si la sociologie générale et les sciences des religions veulent bien accepter quelques fruits de son labeur. En proposant aux problèmes généraux ses solutions particulières, elle n'incite pas à généraliser, à extrapoler, mais elle suggère méthodes et vraisemblances.

Déjà des savants appliqués à l'étude des religions lui ont fait confiance : "C'est seulement dans la sociologie qui prend à son tour pour matière les données de la science comparative des religions que le facteur religieux sera entièrement défini et expliqué par la société et c'est seulement par la sociologie que la science des religions pourra être intégrée à l'ensemble des sciences sociales "(61). La déclaration de deux des historiens les plus qualifiés des sciences religieuses a été souscrite par de nombreux savants qui, livrant les résultats de leurs recherches sur une croyance ou une liturgie, invitent le sociologue à prolonger leur fructueux effort (62).

$\mathrm{Au}$ vrai, beaucoup de ces historiens sont eux-mêmes des sociologues et c'est leur intelligence des représentations et des forces collectives, de la vie totale des groupes humains, du réseau des relations organiques, affectives, qui les a rendus maîtres de tant d'énigmes apparentes des croyances, des cultes et des disciplines.

(60) Oserai-je insister sur ce qu'une sage psychologie des angoisses collectives suggère, dans l'étude de toute société. Comment notre génération, torturée par la peur, regarderait-elle les invasions et les massacres du passé comme simples sujets de littérature ? Fromm (1951), Jones (1951) indiquent des pistes.

(61) Puech et Vignaux, dressant le bilan de la Science des religions dans un tableau des Sciences sociales en France (1937).

(62) Ainsi, P.M. Schunu, Essai sur la formation de la pensée grecque, préface à la seconde Edition, Paris, 1949. - Et, pour le catholicisme, Mgr ANDRIEv, Les Ordines romani du haut moyen age. II. Les textes, Louvain, 1948, pp. xIII-xIv. Dans la Revre des sciences religieuses, 1948, P. Nedoncelde invite l'historien du dogme à suivre l'évolution populaire, sociale, des mots (Prosopon et persona, pp. 277-299) et L. BouYER, pp. 313-333, montre dans la théologie du corps mystique une "surnaturelle creation sociologique n.

Ces quatre appels nous viennent de la seule Université de Strasbourg. Nous pourrions tourner notre oreille vers beaucoup d'autres points de l'horizon, pour entendre les échos.

Schóllgen (1953) et après lui Geck ont discuté les rapports entre sociologie religieuse et morale catholique. 


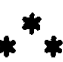

De leur côté, les sociologues de toutes catégories trouvent dans nos enquêtes une réponse partielle à leurs vastes interrogations.

Individu et société : où voit-on mieux que dans les religions l'incorporation mortifiante en vue du salut personnel, la différenciation des statuts et la spécialité des offices au service de l'unité ; la constitution de groupes légaux ou volontaires? Remontons jusqu'aux sources de l'être : où voit-on mieux la notion même du groupe et sa nature, la concurrence du charisme et de l'autorité, le seuil infranchissable de la personne ? Le mystique a fermé sa porte, le sociologue n'explorera que son environnement. Il connaît ses frontières, ses limites. Mais dans son royaume, les structures - hiérarchies, compagnies - brillent au sein des cités et des champs dont la sociologie, urbaine ou rurale, cherche à percer les ombres.

Ontologie, psychologie sociale, nous avons rencontré dans ce rapide itinéraire tous les districts de la science, et les dominant tous, l'épistémologie. La sociologie religieuse n'a point l'outrecuidante prétention d'élever au-dessus de toutes les autres branches de la sociologie un dôme orgueilleux : elle a peu d'illusion sur la proximité du ciel. Mais la nature lui donne des dimensions exceptionnelles et un nombre incalculable de sujets puisqu'elle embrasse tous les hommes et tous les dieux.

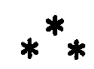

Si les frontières sont indécises ou abaissées entre les sciences des religions et du côté de leurs auxiliaires profanes, une barrière bien marquée sépare ce condominium des sciences appliquées qui l'exploitent. L'effort de science pure unit souvent aux chercheurs désintéressés ceux qui n'ont en vue que cette exploitation légitime. Et les chercheurs, les rallient parfois au-delà des frontières de la science pure. Ce qu'il nous faut marquer ici d'un trait ferme, c'est la distance entre la science pure de la sociologie religieuse et les sciences appliquées qui la prolongent : en les excluant du programme de cette revue, nous n'en avons point contesté l'existence légitime et l'honorable servitude.

Considérons d'abord la science du gouvernement et de l'administration des groupes religieux - grandes Eglises ou communautés locales. Une conduite intelligente suppose adaptation de la société religieuse à la société civile c'est-à-dire connaissance méthodique des structures et de la vie du monde profane pour le modelage des cadres et l'inspiration des méthodes dans le monde représentatif du sacré. Cette nécessité, la hiérarchie catholique n'est pas seule à la comprendre. Elle préoccupe les autres confessions chrétiennes (63). Elle n'échappe point aux musulmans

(63) Il est naturel que l'intérêt des catholiques militants, clercs ou laïs, ait pour cause le désir d'un apostolat lucide : ils ne cessent de justifier leurs recherches pour convaincre ou desarmer les sceptiques. Mêmes préoccupations dans le protestantisme (Leenhardt, Ellul). Des bilans périodiques des travaux accomplis en France par Economie et IItumanisme (R. P. LeBreT), par le C.P.M.I. (R. P. Motre), par M. le chanoine Boulard, par J. Labbe.ss, permettent de 
lucides, qui perçoivent l'ébranlement que la civilisation contemporaine inflige aux cinq piliers de l'Islam ni aux bikkhus lettrés qu'inquiète l'avenir du bouddhisme. Dans le monde chrétien, elle aboutit à un monceau d'utiles statistiques et de graphiques commentés. Les autres religions ne s'enrichissent que d'analyses et de pronostics.

La science politique serait encline à regarder la sociologie religieuse comme un de ses départements. Tous les Etats ne tiennent-ils point les religions pour une des forces qui les étayent ou les menacent ? Evidente en Europe, où le christianisme, jadis ferment de l'unité est devenu le soutien ou l'ennemi des républiques, cette vérité illumine toute l'histoire de l'Islam. Quand le religieux et le profane s'unissent étroitement, la sociologie religieuse est en même temps une sociologie politique. Quand ils se séparent, comme dans la France d'aujourd'hui, le jeu consiste à les accorder. L'un des problèmes de tous les Etats contemporains n'est-il point de délimiter la société religieuse, qu'il désire son dépérissement, comme dans les démocraties populaires ou son cantonnement comme en Egypte (64).

Il nous faudrait reprendre tous les chapitres des relations entre les deux mondes terrestres, le religieux et le profane pour relcver tous les intérêts qu'offre à un Etat la connaissance des sociétés religieuses : leurs ressources matérielles, leurs attitudes familiales, leurs usages économiques (65).

Les partis, eux aussi, tiennent compte pour leur propagande, favorable ou hostile, des données de la sociologie religieuse. Ce qui explique les réserves du Saint-Siège et de certains évêques, craintifs de l'utilisation de renseignements propres à encourager ou à éclairer des actions nuisibles à l'Eglise (66).

Ajouterai-je que les méthodes mêmes de la sociologie religieuse ont été transposées au profit de la politique pure? (67).

Le mouvement des sociétés religieuses n'est pas indifférent aux divers corps de métier qui en éprouvent les oscillations. Certes, ils peuvent se borner à des prévisions élémentaires : mais le commerce, les transports, l'urbanisme tireraient parti d'une vue moins limitée (68).

suivre les progrès d'équipes très actives, groupées dans un Centre catholique dont le siège est 282, rue Saint-Honoré. Tous les pays catholiques rivalisent aujourd'hui pour la construction d'une sociologie pastorale qui a déjà ses premiers manuels (Banning).

(64) Une politique d'hostilité ou de compétition - et les exemples sont nombreux dans le passé comme dans le présent-suppose une connaissance approfondie des sociétés religieuses. Beaucoup de gouvernements dans le passé échouèrent par ignorance. L'expérience peut porter sur un secteur limité de la puissance ecclésiastique, ainsi la juridiction, dans l'Egypte contemporaine.

(65) Problèmes classiques de l'influence des diverses religions sur la fréquence, la fermeté, la fécondité des mariages; sur l'alimentation, le commerce, la répartition des biens. Démographes et economistes, encouragent l'effort des sociologues. La grande dispute sur la part du protestantisme dans l'avènement du capitalisme se prolonge et s'amplifie.

(66) Les réserves de la hiérarchie en Italie, en Belgique, portaient sur la convenance de la publication des résultats.

(67) Mon collègue Ossowski m'a assuré qu'elles ont servi a établir des critères de discrimination profane dans les régions recouvrées par la Pologne.

(68) Voyez le chapitre Ecologie dans La sociologie au $X X^{\circ}$ siecle. 


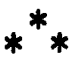

Nous avons justifié notre résolution par le besoin, les moyens et les fins. Elle ne se justifiera pleinement que par l'efficience.

Conforme au modèle annoncé, le premier numéro se divise en trois parties. En honorant Joachim Wach, nous traduisons l'amitié qui animait plusieurs d'entre nous à cet homme cordial et généreux, mais surtout notre admiration pour son œuvre et notre estime pour sa méthode large qu'il tentait d'élargir encore quand la mort l'a emporté (69). Notre dernier entretien nous assure qu'il eût pris intérêt au mémoire de notre ancien élève Jacques Petit, dont beaucoup de personnes réclamaient la publication puisqu'il inaugure une méthode d'enquête plusieurs centaines ou plusieurs milliers de fois pratiquée: exemple curieux du succès d'un manuscrit unique et de bibliothèque privée (70).

Faute de place, nous avons dû ajourner la publication de plusieurs notes sur l'état de la sociologie religieuse en différents pays; nous n'avons conservé sous la rubrique Notes et documents que trois articles sur les sept qui nous étaient proposés.

Enfin, la nomenclature des thèses et diplômes français relatifs à la sociologie religieuse, la bibliographie récente, la critique des ouvrages feront ressentir la vitalité de notre science.

Aux prochains numéros, des articles sur le bouddhisme, l'islamisme et les religions africaines élargiront encore notre horizon que nous voulons étendre aux dernières limites du temps et de l'espace. Tous ceux qui croient à l'avenir des sciences religieuses, dont la sociologie est comme un réceptacle, mieux encore un foyer, nous seconderont dans une tâche que récompensera, si nous le méritons, l'élévation de la culture et s'il plaît aux dieux, un progrès de la sagesse humaine.

Gabriel LE Bras,
Professeur à l'Université de Paris,
Président honoraire
de la Section des Sciences religieuses
de l'Ecole pratique des Hautes Etudes.

(69) Il nous disait son intention de donner à l'histoire et aux pratiques une place accrue dans ses recherches.

(70) F. Isambert a bien voulu préparer l'édition abrégée de ce document original. 